\title{
Molecular fluorescence of $\mathrm{CaOH}, \mathrm{SrOH}$ and $\mathrm{BaCl}$ in flames
}

\author{
H. G. C. HumaN* and P. J. Th. ZeEgers \\ Fysisch Laboratorium, Rijksuniversiteit, Utrecht, The Netherlands
}

(Received 12 September 1974)

\begin{abstract}
Molecular fluorescence of $\mathrm{CaOH}, \mathrm{SrOH}$ and $\mathrm{BaCl}$ was observed in a hydrogen-oxygenargon flame, using a continuum source of excitation. Fluorescence and emission spectra were identical. The spectral distribution of the fluorescent light within a band is independent of the wavelength distribution of the irradiating light, indicating that the absorbed energy is redistributed before the fluorescence process. It was found, using wideband filters in the irradiating light beam, that energy is exchanged between the different electronic levels. The fluorescence efficiencies were determined and found to be of the same order of magnitude as the atomic flourescence efficiencies.
\end{abstract}

\section{INTRODUCTION}

The ExisTeNce of molecular absorption bands of $\mathrm{CaOH}$ around $5540 \AA$ which interfere with atomic absorption measurements of barium at $5535 \AA$ is well known [1-3]. Similarly, the $\mathrm{SrOH}$ bands in the $6700 \AA$ region may cause a spectral interference with the lithium $6705 \AA$ line [1]. Other examples of molecular absorption occurring in flames used for analytical atomic absorption or emission measurements are known, e.g. absorption bands of the alkali halides in low temperature flames [1] and absorption bands of nitrogen, carbon and sulphur compounds in flames of higher temperature $[4,5]$.

It is reasonable to expect, therefore, that it should be possible to observe molecular fluorescence of these substances in flames if a continuum source of high intensity is used as irradiating source. Such fluorescence, viz. that of $\mathrm{CaOH}$, was indeed first reported at the Second International Atomic Absorption and Fluorescence Conference [6]. Results are presented in this paper of measurements made to obtain more information on the fluorescence of alkaline earth salts in flames.

\section{Expertmental}

The primary irradiating source used was a $150 \mathrm{~W}$ xenon are enclosed in a parabolic reflector (the Eimac lamp). This lamp yields approximately 10 times more flux than a conventional xenon arc as a result of the large solid angle over which the radiation is collected. A disadvantage of this source is instability, which probably is the result of are wander. A mochanical chopper was used to modulate the primary radiation beam at $50 \mathrm{~Hz}$.

An argon-hydrogen-oxygen shielded flame was used as the atom or molecule

* Present address: National Physical Research Laboratory, C.S.I.R., P.O. Box 395, Pretoria 0001, South Africa.

[1] S. R. Koirtyohans and E. E. PiCketr, Anal. Chem. 38, 585 (1966).

[2] L. Capacho-Delqado and S. Spraque, Atomic Absorption. Newsletter 4, 363 (1965).

[3] S. R. Koirtyohans and E. E. Pickett, Anal. Chem. 38, 1087 (1966).

[4] K. Fuwa and B. L. Valleie, Anal. Chem. 41, 188 (1969).

[5] J. A. Fronino, R. N. KNISWLEy and V. A. FAsser, Spectrochim. Acta 28B, 413 (1968).

[6] D. R. Jenknvs, 2nd Int. At. Abs. and Fluorescence Conf., Sheffield (1969). 
reservoir. It consisted of an inner flame of $10 \mathrm{~mm}$ diameter into which the solution aerosol could be sprayed, and an outer surrounding flame of the same gas composition to shield the inner flame from atmospheric gases and to provide a homogeneous temperature across the inner flame. A fuel-rich flame with a temperature of $1880 \mathrm{~K}$ was found to yield the strongest molecular fluorescence signals. As the temperature of the flame decreased, the signal-noise ratio increased, the noise being caused by thermal emission of the molecules in the flame which was much stronger than the fluorescent emission. A lower limit to the temperature is set by reflection of the incident light beam into the monochromator by unevaporated salt particles. This effect increases sharply with flame temperatures lower than $1900 \mathrm{~K}$. A stoichiometric flame with a temperature of $2140 \mathrm{~K}$ was also used. The gas flows and calculated composition of burnt gases of the two flames are shown in Table 1.

Table 1. Composition of flames

\begin{tabular}{llccc}
\hline \multirow{2}{*}{ Gas } & \multicolumn{2}{c}{ Fuel-rich $(\mathrm{T}=1880 \mathrm{~K})$} & Stoichiometric $(\mathrm{T}=2142 \mathrm{~K})$ \\
\cline { 2 - 5 } & flow, 1/min & partial pressure & flow, $1 / \mathrm{min}$ & partial pressure \\
\hline $\mathrm{Ar}$ & 6.5 & 0.756 & 5 & 0.712 \\
$\mathrm{O}_{2}$ & 0.87 & $<0.001$ & 1. & 0.002 \\
$\mathrm{H}_{2}$ & 2.1 & 0.042 & 2 & 0.005 \\
$\mathrm{H}_{2} \mathrm{O}$ & & 0.202 & & 0.278 \\
\hline
\end{tabular}

The height of measurement in the flame was not critical as long as the excessively radiating primary reaction zone was avoided. A slit width of $0.15 \mathrm{~mm}$ (spectral bandwidth of approx. $6 \AA$ ) and slit height of $4 \mathrm{~mm}$ were used. The concentrations of the salt solutions sprayed into the flame were usually $1 \%$ (weight per volume).

\section{Results}

\subsection{Spectra obtained}

Figure 1 shows the emission (top) and fluorescence (bottom) spectra of $\mathrm{CaOH}$ in the flame. Figure 2 shows the same for SrOH. The similarity between the fluorescence and emission band intensities is striking. For $\mathrm{CaOH}$, the two main bands with maxima at $5540 \AA$ and $6230 \AA$ are believed to originate from different electronic configurations [7]. The maxima at $6033 \AA$ and $6430 \AA$ are believed to originate from the same electronic configuration as the band maximum at $6230 \AA-$ they only have different vibrational energy levels [8]. The maxima at 5525 $\AA$, $5540 \AA$ and $5725 \AA$ are similarly due to transitions between different vibrational levels of the same electronic configuration.

[7] C. G. JAMres and T. M. Sugper, Nature 175, 333 (1955).

[8] G. A. J. Momlands, Internal Report No. V3694, State University, Utrecht. 


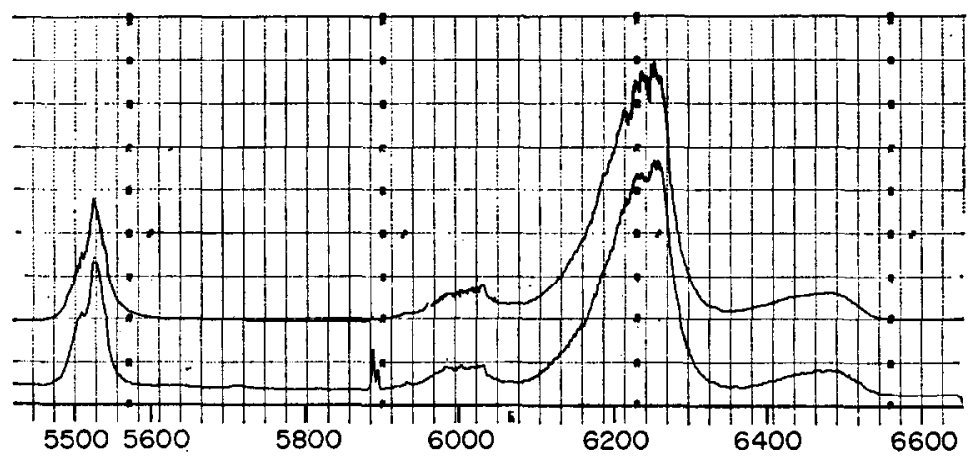

Fig. 1. Emission (top) and fluorescence (bottom) spectra of $\mathrm{CaOH}$. Horizontal scale: wavelength in $\AA$.

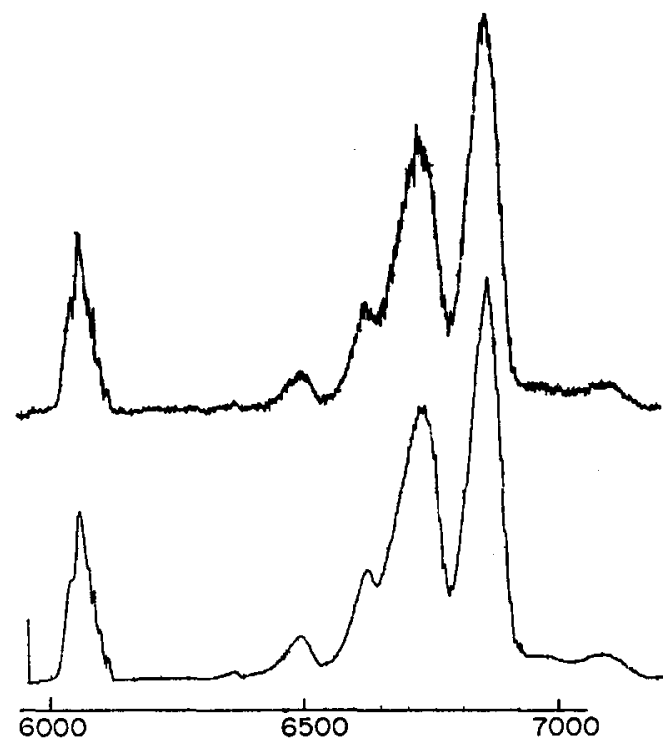

Fig. 2. Emission (bottom) and fluorescence (top) spectra of SrOH. Horizontal scale: wavelength in $\AA$.

Figure 3 shows the fluorescence spectrum when a solution of $\mathrm{BaCl}_{2}$ is sprayed into the flame. According to Herzberg [15] this spectrum has to be ascribed to barium monochloride, $\mathrm{BaCl} . *$ The $\mathrm{BaO}$ bands between $4500 \AA$ and $7900 \AA$ and

* Also the relatively narrow bands in comparison with the $\mathrm{CaOH}$ and $\mathrm{SrOH}$ bands point to a diatomic emitter.

[9] S. J. Pearce, L. de Galan and J. D. Winetordnhr, Spectrochim. Acta 23B, 793 (1968).

[10] P. J. Th ZmHagrs and J. D. Winteordner, Spectrochim. Acta 26B, 161 (1971).

[11] D. R. JHNkTNs, Trans. Faraday Soc. 64, 38 (1968).

[12] M. KasHa, J. Opt. Soc. Amer. 88, 929 (1948).

[13] J. C. DF Vos, Thesis, University of Amsterdam (1953).

[14] T. J. Hollander, P. L. LiJnse, L. P. L. Franken, B. J. Janshen and P. J. Th. Zgerghes, J. Quant. Spectry. Radiative Transfer 12, 1067 (1972).

[15] G. HerzzBerg, Molecular Spectra and Molecular Structure-I. Spectra of Diatomic Molecules, 2nd edn. Van Nostrand, Princeton (1950). 


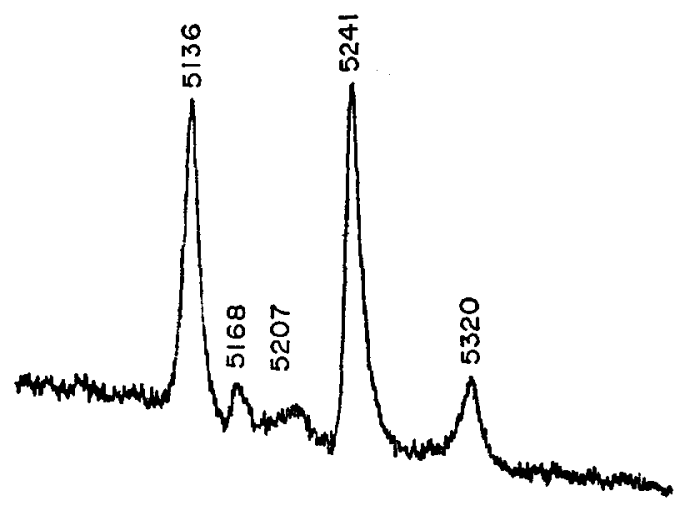

Fig. 3. Fluorescence spectrum of BaCl. Approximate wavelengths of peaks are denoted in $A$.

the green $\mathrm{BaOH}$ bands with maxima at $4870 \AA$ and $5120 \AA$ which emit strongly in the flame could not be observed in fluorescence, from which it seems improbable that the lower electronic levels involved are ground state levels.

\subsection{Spectral distribution of fluorescent light}

Using appropriate wideband filters, absorbing the irradiating light around $6230 \AA$ and $5540 \AA$ in turn, it was found for $\mathrm{CaOH}$ that light absorbed at $6230 \AA$ was radiated as fluorescent light at $5540 \AA$, and vice versa, viz. fluorescence at $6230 \AA$ as a result of absorption at $5540 \AA$.

The only filter available for $\mathrm{SrOH}$ was one with high transmission at the wavelength of the $6860 \AA$ bandhead and low transmission at the bandhead at $6060 \AA$. The same phenomenon as with $\mathrm{CaOH}$ was observed, viz. strong fluorescence at $6060 \AA$ as a result of absorption at higher wavelengths, as the results in Table 2 show.

Table 2. Ratio of fluorescence signals with and without filter in irradiating beam, for SrOH

\begin{tabular}{ccc}
\hline Wavelength in $\AA$ & 6860 & 6060 \\
\hline $\begin{array}{c}\text { Ratio } \\
\begin{array}{c}\text { Transmission factor } \\
\text { of filter }\end{array}\end{array}$ & 0.72 & 0.64 \\
\hline
\end{tabular}

By using an interference filter in the irradiating light beam between the lamp and flame, the continuum was reduced to a band with halfwidth of approximately $180 \AA$. At normal incidence the centre frequency was approximately $100 \AA$ above the $\mathrm{CaOH}$ band maximum at $6230 \AA$, and when the filter was used at a certain inclination to the optical axis the centre frequency was approximately $100 \AA$ below $6230 \AA$. The spectral distribution of the fluorescence band was measured with and without a filter in the irradiating beam by scanning with the monochromator using 
a narrow entrance slit. Figure 4 shows the results of measurements with the filter centre frequency $100 \AA$ below the band maximum. It is clearly seen that the spectral distribution of the fluorescent light is the same with and without the filter in the irradiating beam. Similar results were obtained by using the filter at normal incidence, i.e., with centre frequency $100 \AA$ above the band maximum. This experiment then proved that after absorption of energy at a certain wavelength in the band, this energy is redistributed among the vibrational and rotational levels before reradiation as fluorescent light. Whether the absorbed energy is divided between the heads at $6230 \AA, 6033 \AA$ and $6430 \AA$ in the same proportions with and without selective irradiation of the flame could not be ascertained as a result of the low intensities of the side bands.

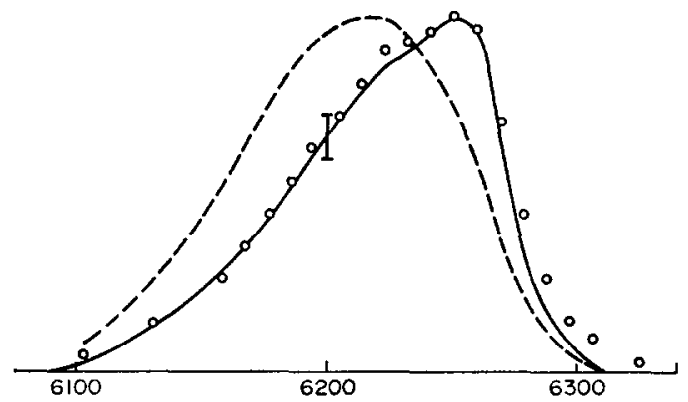

Fig. 4. Intensity distribution of $6230 \AA$ band of $\mathrm{CaOH}$. The circles represent the measured spectral distribution of the $6230 \AA$ band without any filter in the beam. The solid line represents the measured distribution with the filter between primary source and flame. The broken line is the distribution of the band intensity, taking the transmission characteristics of the filter into account, i.e. this distribution would have been measured with the filter in front of the monochromator alit. Horizontal scale: wavelength in $\AA$.

\subsection{Fluorescence efficiencies}

The fluorescence efficiency is defined as the ratio of the energy appearing as fluorescent radiation and the absorbed energy. It is in practice obtained from the relation

where $Y=$ fluorescence efficiency

$$
Y=\frac{I_{F}}{I_{A}} \cdot \frac{4 \pi}{\omega} \cdot \frac{G}{A}
$$

$I_{F} \quad=$ measured fluorescence signal

$I_{A}=$ measured absorption signal

$4 \pi / \omega=\mathrm{a}$ factor to take into account the finite solid angle over which fluorescence radiation is collected

$A \quad=$ a factor to account for the different amplification factors when measuring fluorescence and absorption signals

$G=$ geometrical factor to account for the differences of volumes in the flame that absorb and fluoresce $[9,10]$

Attempts were made to determine the fluorescence efficiencies of the molecular fluorescence of $\mathrm{CaOH}$. Since energy is exchanged between the two electronic levels, 
nine $Y$ values are involved [11], e.g., $Y_{1-2}$, the efficiency of fluorescence from level 2 as a result of absorption to level $1 ; Y_{1.2-8}$, the efficiency of fluorescence from level 2 as a result of absorption to levels 1 and 2. The factors $Y_{1-1}, Y_{2-2}, Y_{1,2-1}, Y_{1,2-1,2}$, $Y_{2-1,2}$ and $Y_{1-1,2}$ are obtained in similar ways. The fluorescence signals of both bands had therefore to be measured while blanking out irradiation at the two wavelengths in turn with filters. Assuming that the radiation at the bandheads of $5540 \AA$ and $6230 \AA$ are representative for the whole bands, the fluorescence and absorption were measured with narrow slits at the band maxima. This assumption is partially justified for the $6230 \AA$ band by the results obtained with the interference filter, showing that energy is redistributed before fluorescence occurs within the $6230 \AA$ band. A glass filter with high transmission at $5540 \AA$ and low transmission at $6230 \AA$ was available. A liquid filter containing equal amounts of $\mathrm{CuSO}_{4}$ and $\mathrm{NiSO}_{4}$ [12] was made up for obtaining high transmission at $5540 \AA$ and absorption at $6230 \AA$. Table 3 shows the measured transmission values at the two wavelengths.

Table 3. Transmission of filters

\begin{tabular}{ccc}
\hline Wavelength & Glass filter $T_{1}$ & Liquid filter. $T_{2}$ \\
\hline 5540 & $0.2 \%$ & $43.6 \%$ \\
6230 & $69.3 \%$ & $1.3 \%$ \\
\hline
\end{tabular}

The instability of the xenon arc made exact measurements of the small absorption signals ( $\sim 1 \%$ absorption) very difficult. This problem was overcome by measuring the percentage absorption signal with another continuum lamp of good stability, viz. a tungsten strip filament lamp. The product of this percentage absorption and the intensity of the xenon are then gives the magnitude of the absorption signal, assuming that the percentage absorptions of the xenon arc and the filament lamp are equal.

Table 4 gives the measured values of the fluorescence efficiency for the fuel-rich and stoichiometric flames. The relative spectral sensitivity of the measuring apparatus (photomultiplier and monochromator) at the two wavelengths was determined using a calibrated tungsten strip lamp run at $7 \mathrm{~A}$ as source and using the radiance

Table 4. Fluorescence yield factors of $\mathrm{CaOH}$ for a fuel-rich and a stoichiometric flame

\begin{tabular}{cllll}
\hline & $Y_{1-1}$ & $Y_{1-2}$ & $Y_{2-1}$ & $Y_{2-2}$ \\
\hline fuel-rich flame & 0.01 & 0.02 & 0.03 & 0.09 \\
stoichiometric flame & 0.015 & 0.03 & 0.05 & 0.14 \\
\hline
\end{tabular}


values of $\mathrm{DE}$ Vos [13]. The intensities of absorption and fluorescence were determined in relative numbers of photons. The level from which the $5540 \AA$ radiation originates is termed level 1 and the lower-lying level of the $6230 \AA$ radiation, level 2.

\section{Discussion}

A study of molecular fluorescence may yield interesting information on the interaction between molecules in flame gases. In fact, just as atomic fluorescence studies elucidate atom-molecule interactions, a wealth of information can be extracted from molecular fluorescence data. These first measurements of fluorescence efficiencies of the alkaline earth hydroxides in flames showed that they are of comparable magnitude to atomic fluorescence efficiencies [14].

Due to the instability of the primary source the accuracy of measurement is not high. However, the $Y$-values reported should be considered as approximate figures only, because the situation may be much more complex than the assumed two-level model. For example, it is quite possible that the energy transfer to the $5540 \AA$ band from the $6430 \AA$ band is different from that of the $6230 \AA$ band, etc. Furthermore, the bands with heads at $5525 \AA$ and $5540 \AA$ overlap to an unknown degree. The yet unexplained fine structure of the bands makes interpretation of fluorescence efficiencies very difficult.

On the analytical side, molecular fluorescence may possibly be applied to the analysis of non-metallic elements such as sulphur, phosphorus, carbon, nitrogen and the halogens, by measuring the fluorescent radiation of the compounds of these elements. These bands often lie in the visible or ultraviolet, whereas their atomic resonance lines are in the far or vacuum ultraviolet which makes measurement more difficult. The sulphur dioxide bands, for instance, exhibit a maximum absorption at $2100 \AA$ and have already been used for the determination of sulphur in amino acids and proteins by the absorption method [4]. The experience with $\mathrm{CaOH}$ showed that the fluorescence signals are in practice easier to detect and measure than the absorption spectra if a continuum lamp with high radiation power is available.

As with atomic fluorescence measurements using conventional sources, the primary light source remains the limitation of the technique. A tunable dye laser would solve many problems. If the bandwidth of radiation could for instance be adjusted to be of the same halfwidth as that of the $\mathrm{CaOH}$ band at $6230 \AA$, fluorescence at any of the other bandheads could be detected and the yield factors determined without the use of filters. In analytical applications using a dye laser, the problem of scattering of light from the primary source into the detector can be eliminated by measuring fluorescence at a wavelength not radiated by the laser, if the fluorescence yield at wavelengths other than the absorption wavelength is as high as it is for $\mathrm{CaOH}$ and $\mathrm{SrOH}$. 